\title{
Handedness measures for the Human Connectome Project: Implications for data analysis
}

Lana Ruck ${ }^{1,2,3}$, P. Thomas Schoenemann ${ }^{1,2,3}$

${ }^{1}$ Cognitive Science Program, Indiana University, Bloomington IN, USA

${ }^{2}$ Department of Anthropology, Indiana University, Bloomington IN, USA

${ }^{3}$ Stone Age Institute, 1392 W Dittemore Road, Gosport, Indiana, USA

\author{
Corresponding Author: \\ Lana Ruck \\ Department of Anthropology \\ Indiana University \\ 701 E. Kirkwood Ave, SB 166 \\ Bloomington, IN 47405 \\ lruck@iu.edu; 812-606-4962 \\ ORCiD: https://orcid.org/0000-0001-6067-8735
}




\begin{abstract}
Open data initiatives such as the UK Biobank and Human Connectome Project provide researchers with access to neuroimaging, genetic, and other data for large samples of left-and righthanded participants, allowing for more robust investigations of handedness than ever before. Handedness inventories are universal tools for assessing participant handedness in these largescale neuroimaging contexts. These self-report measures are typically used to screen and recruit subjects, but they are also widely used as variables in statistical analyses of fMRI and other data. Recent investigations into the validity of handedness inventories, however, suggest that self-report data from these inventories might not reflect hand preference/performance as faithfully as previously thought. Using data from the Human Connectome Project, we assessed correspondence between three handedness measures - the Edinburgh Handedness Inventory (EHI), the Rolyan 9hole pegboard, and grip strength - in 1179 healthy subjects. We show poor association between the different handedness measures, with roughly $10 \%$ of the sample having at least one behavioral measure which indicates hand-performance bias opposite to the EHI score, and over $65 \%$ of lefthanders having one or more mismatched handedness scores. We discuss implications for future work, urging researchers to critically consider direction, degree, and consistency of handedness in their data.
\end{abstract}

\title{
Keywords
}

Handedness; Edinburgh Handedness Inventory; Measurement; Laterality; Neuroimaging;

\section{Word Count}

7065 main text; 10104 all 


\section{Introduction and motivation}

Since it was first published in 1971, the Edinburgh Handedness Inventory (henceforth EHI; Oldfield, 1971) has become the gold-standard for evaluating handedness in neuropsychological contexts. The use of some version of the EHI is now ubiquitous for subject screening and participant exclusion in neuroimaging studies, often to the omission of other methods of handedness assessment (Fazio \& Cantor, 2015). The exclusion of left-handed subjects (defined as those with either low or negative EHI scores) in neuroimaging contexts-specifically those

8 probing language or other lateralized functions - is largely justified, considering the effect of

9 subject lateralization on variance in activation patterns and resultant issues with common statistical

10 approaches (see Bailey, McMillan, \& Newman, 2019; Króliczak, Gonzalez, \& Carey, 2019;

11 Vingerhoets, 2014). Still, some have argued that this is a misguided approach, claiming that the

12 inclusion of left-handers and other atypically lateralized individuals can tell us more, not less,

13 about cortical function, and especially, asymmetry (Willems et al., 2014). Unfortunately, after

14 decades of research paradigms which purposefully included left-handers, handedness effects on

15 other aspects of laterality are still poorly understood. Efforts to identify a coherent relationship

16 between handedness and language laterality, for example, have proceeded for over a century, and

17 yet still, many studies show contrasting relationships between subject handedness and hemispheric

18 activation patterns (Badzakova-Trajkov et al., 2016; Mazoyer et al., 2014; Mellet et al., 2014;

19 Somers et al., 2015; Zago et al., 2016).

Disparate findings from large-scale studies of functional brain lateralization and

21 handedness have resulted in revived discussions - at least from within the community of laterality

22 researchers - on the use of the EHI and other handedness inventories. One result of these renewed

23 discussions is a "degree vs. direction" approach, where subjects are classed not into binary

24 directional categories (right- vs. left-hander), but into ordinal categories reflecting degree of 
25 handedness, such as strong right-handed, mixed-handed, weak left-handed, etc., prior to analysis

26 (Gonzalez \& Goodale, 2009; Kaploun \& Abeare, 2010; Newman et al., 2014; Pritchard et al.,

27 2013; Somers et al., 2015). This approach has led to some improvements, most notably in linking

28 variability in left-handers' hand performance, which is generally lacking in right-handers, to

29 similar variability in activation patterns in the brain (Christman et al., 2015). Still, the partition of

30 EHI scores into ordinal categories is arguably a least-effort and post hoc approach to addressing

31 issues with handedness measures and classification, and - despite many critiques - the use of EHI

32 values as a singular handedness assessment remains consistent.

33 Much was done to evaluate the internal consistency and validity of handedness inventories,

34 and to compare various handedness assessments to each other, in the 1970's and 1980's. Overall,

35 however, the expediency of self-report measures like the EHI resulted in their ubiquity over other

36 handedness assessments (especially behavioral ones, which take time and resources to administer).

37 Handedness literature from the past decade is arguably returning to these roots, as more recent

38 discussions parallel earlier debates on handedness assessment and characterization (see Annett,

39 1985; Bryden, 1977; McManus, 1984, 1985, 1986). Despite a revived interest in the validity of

40 handedness inventories (Büsch et al., 2010; Dragovic, 2004; Fazio et al., 2012, 2013; Milenkovich

41 \& Dragovic, 2013; Veale, 2014), to our knowledge, only a few studies have been conducted

42 directly comparing self-report, survey-based handedness classifications with actual measures of

43 hand performance and hand preference (Brown et al., 2004, 2006; Bryden et al., 2011; Corey et

44 al., 2001; Flindall \& Gonzalez, 2018; Gonzalez et al., 2007; Leppanen et al., 2018; McManus, Van

45 Horn, \& Bryden, 2016). Some of these studies evaluated a different handedness inventory as their

46 survey-based measure (such as the Waterloo Handedness Questionnaire, or WHQ), but in general,

47 participants in each of these studies were asked to complete both the survey and behavioral

48 handedness assessments. These tasks typically include: at least one version of a pegboard task 
(Annett, grooved, etc.), finger tapping, grip strength, and other behavioral measures. Raw scores and laterality indices (or LI's, see Methods below) for performance measures are then compared

to the survey score, which is also a laterality quotient (see Edlin et al., 2015, however, for a

52 discussion on EHI administration and scoring inconsistencies; also see McManus, Van Horn, \&

53 Bryden, 2016: 387-388, for a discussion on simple differences between hands versus laterality

54 index calculations).

Brown and colleagues (2004) assessed the correlations between five performance measures

and the WHQ in 62 participants, and found that most measures had significant correspondence to

the WHQ, with the exception of the grooved pegboard. They later (2006) conducted the same

measures on 120 additional subjects, and found the same results - that all measures save for grip

(ranging between $7 \%$ and $49 \%$ ) showed at least one behavioral measure with a different

64 for predicting WHQ with behavioral scores showed the Wathand box test (WBT) as the most

65 significant predictor of WHQ scores, and that grip strength was the only non-significant predictor.

66 In a third study, they assessed the WBT and the WHQ as they related to language laterality in 142

67 subjects, using the Fused Dichotic Words Test (Bryden et al., 2011). They found that both tasks -

68 one behavioral and one survey-based - had non-significant correlations with the language data,

69 with the exception of the WHQ and language laterality in females. They conclude by urging further

70 work comparing behavioral handedness measures to language lateralization, implicating fMRI as

71 a component of future research. 
Corey and colleagues (2001) assessed the correspondence between two performance

73 measures including the EHI, and finger tapping, grip strength, and a grooved pegboard task, in 52

74 healthy subjects. They found high correspondence between preference and performance data, and

75 only 5 subjects showed different handedness classifications between performance LI's and survey

76 classifications (two left-handers and 3 right-handers). Despite this high correspondence, they

77 recommend the use of multiple metrics to classify subjects based on handedness. They conclude:

"Whether other anatomic asymmetries are linked to hand preference or performance or both remains unclear. A multivariate approach to defining handedness and a multivariate examination of anatomic asymmetries may clarify the relationship of handedness to other

Overall, these studies suggest that there are complicated patterns of association even between the

84 different measures of handedness, and that survey-based and behavior-based handedness

85 assessments are likely not isomorphic. These issues, obviously, would complicate any efforts to

86 understand how handedness relates to other human asymmetries (see Gonzalez et al. 2018;

87 Hopkins, 2018, for recent discussions of these topics).

In contrast to these approaches, Gonzalez and colleagues (2009) assessed correspondence

89 between the EHI and hand-performance using more naturalistic tasks - puzzle and LEGOß-

90 building - in 20 subjects. They filmed subjects' hand movements and created LI's for how often

91 subjects used each hand, and then compared these data to EHI scores. They showed high

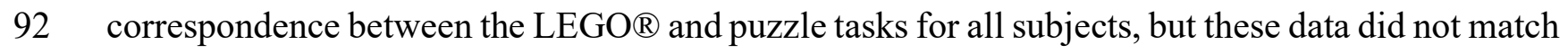

93 well with the EHI scores, especially for left-handers. Although all 10 left-handers in the sample

94 were classed as strong left-handed (average EHI $=-94.1$ ), at least half of them used their right

95 hand more frequently than their left in the behavioral task (Gonzalez et al., 2007: 277). In a later 
study probing language laterality with a dichotic listening task, they assessed correlations between

97 the EHI, grip strength, finger tapping, and the LEGO® task in 36 subjects (Gonzalez \& Goodale,

98 2009). They found that the LEGO ${ }^{\circledR}$ task measures were the only ones with significant correlations

99 with the dichotic listening task. Although they also found significant correspondence between the

100 EHI, grip strength scores, and finger tapping scores, these measures did not correlate with the

101 dichotic listening task language laterality, as the LEGO ${ }^{\circledR}$ task did. They suggest that, in their

102 sample, "there is something about visuomotor control and handedness that does not map onto other

103 measures of laterality in motor control" such as the EHI and more common behavioral tasks

104 (Gonzalez \& Goodale, 2009: 3187).

105 In another recent paper (Flindall \& Gonzalez, 2018), this team combined two handedness

106 inventories, the EHI and Waterloo Handedness Questionnaire (for a combined survey, the E-

107 WHQ) and assessed the survey's accuracy, reliability, and ability to predict grasping patterns. In

108 a meta-analysis of data from their previous studies, Flindall and Gonzalez (2018) showed that \%

109 right hand use for grasp-to-build tasks correlated significantly with E-WHQ survey scores, but

110 only for the entire population. This effect was not replicated when subjects were grouped into

111 subsets by handedness, as all correlations between E-WHQ scores and \% right-hand use in Left,

112 Right-, and Ambidextrous-handers were non-significant; they state that "within self-defined

113 handedness groups, individual score on the E-WHQ is not useful in predicting right-hand

114 preference in a simple grasping task" (Flindall \& Gonzalez, 2018: 7). They also assessed whether

115 or not the consistent Likert design of the survey impacted subject responses, showing that

116 scrambling the response order for the 22-item survey led to significantly lower scores than the

117 traditional test, within the same subjects. Finally, they assessed the consistency of subject

118 responses in a test re-test paradigm, and found that over $90 \%$ of participants changed their response

119 to at least one question. Overall, the team concludes that: “...the accuracy of a single handedness 
121 it comes to predicting hand preference for grasping” (Flindall \& Gonzalez, 2018: 13).

123 handedness-related asymmetries, specifically for language, but extending into other functions as

124 well. Although more naturalistic tasks may be the most viable option, they require more time and

125 effort on the part of the experimenter in terms of recording, coding, and analyzing; this, we feel, 126 explains the ubiquity of survey-based handedness assessments within neuropsychological

127 research, and is one of the largest obstacles to overcome in future work. Aside from the use of

128 more naturalistic manual motor tasks in the studies described above, relatively little has been done

129 to explore the role that the EHI itself, and other handedness measures, may play in obscuring, not

130 elucidating, the complexity of human handedness and its relationship with other phenomena. To

131 add to this discourse, we assess multiple measures of handedness using handedness data from the

132 Human Connectome Project (Van Essen et al., 2013).

\section{Methods}

\section{About the HCP Data}

136 The Human Connectome Project is an open-data initiative which provides structural and

137 functional neuroimaging data to researchers (along with demographic data, and behavioral data

138 for several common psychological assessments) from over 1200 healthy adult participants from

139 the United States. All Human Connectome Project (HCP) subjects complete a battery of tasks,

140 three of which are assessments of participant handedness ${ }^{1}$. The first is the 10-question, or "short

\footnotetext{
${ }^{1}$ Some HCP data, including information about participant handedness, is considered "restricted" by the HCP, meaning that researchers must complete paperwork and request permission in order to gain access to that information. Information on restricted access for the HCP data can be found at https://www.humanconnectome.org/study/hcpyoung-adult/document/restricted-data-usage
} 
141 form" version, of the Edinburgh Handedness Inventory (or EHI, see Oldfield, 1971), which is a

142 standard self-reported survey of hand-use preference for various tasks, and the other two are hand

143 motor measures from the NIH toolbox (Gershon et al., 2010; Kallen et al., 2012a, 2012b; Reuben

144 et al., 2013; see also Wang et al., 2013). These measures include the 9-hole Pegboard Dexterity

145 Test (or Rolyan pegboard) which measures time taken to complete a peg-manipulation task, in

146 seconds (lower values reflect better performance); and a grip strength task, measured in pounds

147 using a dynamometer (higher values mean better performance). Importantly, subjects complete the

148 pegboard and grip strength tasks with both the left- and the right-hand, thus providing behavioral

149 metrics for comparing the laterality of hand motor skills to the self-reported data from the EHI. In

150 addition to these three metrics, the NIH toolbox includes a direct question: "Are you right-handed

151 or left-handed?" - with possible responses being: "Right-handed" "Left-handed" and "Not sure"

152 - so we treat these responses as a participant's self-identified handedness. As subject recruitment

153 was conducted irrespective of handedness, the number of left-handers in this sample, as classed by

154 the EHI ( $n=112$, roughly 10\%), corresponds well with broad handedness-trends across living

155 human populations. Thus, we feel that this is a reliable sample for assessing correspondence

156 between self-reported EHI values and direct physical measures of manual motor skill.

157 Furthermore, with the impressive amount of behavioral and neuroimaging data also provided for

158 these subjects (Barch et al., 2013), the HCP sample provides many future avenues for assessing

159 handedness-related differences in psychometric data, brain anatomy, and brain function.

160 The HCP has released data for over 1200 subjects, but this sample includes several twin,

161 sibling, and parent-child pairs. As we did not exclude for family or twin status in this study, it is

162 important to note that roughly 300 of the $1200 \mathrm{HCP}$ participants are related to at least one other

163 participant. These data present a unique opportunity to study family relationships in future work,

164 and indeed there are undoubtedly many interesting interactions to study between age, sex, twin 
165 status, and handedness in this data set. As effects of sex, age, and other demographics may be as

166 related to the HCP's recruitment and screening procedures as to a true signal, we will focus solely

167 on the handedness measures in this paper, save for our discussion of the Freesurfer (brain) data.

168 Of the $1200 \mathrm{HCP}$ participants, those with positive illicit-drug test results (restricted-access ${ }^{1}$

169 demographic information) were first removed from the sample. Those with either the grip strength

170 or pegboard measures three standard deviations above or below the sample mean were also

171 removed from analysis. Thus, this study includes 1179 of the $1200 \mathrm{HCP}$ subjects. Some of these

1721179 participants did not undergo neuroimaging, and do not have Freesurfer data (see the section

173 on Subject classification in neuroimaging contexts), so our sample for the Freesurfer data is 1096

174 participants. In addition, $46 \mathrm{HCP}$ participants underwent a full test-retest paradigm and have two

175 sets of data to compare for internal consistency of the HCP protocols; one of the test-retest

176 participants was stripped from the larger sample due to outlying handedness scores, so our test-

177 rest data includes 45 participants.

178

Measures and terminology

180 Our main goal is to explore the typical ways in which neuroimagers partition left- and right-

181 handers into groups for analysis. Thus, many of our analyses are run on the entire HCP sample as

182 well as on the right- and left-handed subject subsets, as defined by EHI scores, to mirror the

183 approach of others working with handedness data in neuroimaging contexts. Analyses done on

184 EHI-delineated 'right-handers' $(\mathrm{EHI}>0)$ will be indicated by the term $\mathbf{E H I}+$ and those on EHI-

185 delineated 'left-handers' $(\mathrm{EHI}<0)$ will be denoted $\mathbf{E H I}-$. To reiterate, these terms reflect subject

186 groupings based on EHI scores, and are independent of the behavioral handedness measures for

187 the right- or left-hand, and of the self-identification groupings, which will be indicated as such.

188 We report summary statistics on the EHI and raw grip strength and pegboard scores across all 1179 
subjects, as well as 'left-handers' (EHI- $n=112)$ and 'right-handers' (EHI+ $n=1067)$ separately,

190 in Table 1. Based on the self-identification data alone, discrepancies exist between handedness

191 classification based on EHI scores and subject self-reporting: 120 subjects self-identified as left-

192 handed, 1045 self-identified as right-handed, and 14 self-identified as not sure. As with previous

193 studies (Flindall \& Gonzalez, 2018; Mazoyer et al., 2014), several $(n=25)$ HCP participants with

194 “moderately" right-handed EHI scores (EHI+ participants) self-identified as left handed, and a

195 small number $(n=3)$ of participants with left-handed EHI scores (EHI- participants) identified as

196 right-handed in the HCP sample as well.

197 Due to our particular interest in whether the EHI accurately reflects handedness when

198 measured in other domains, we calculated laterality indices (henceforth, LI or LI's) for the

199 behavioral tasks, using the following approach:

1) Grip strength LI $=\frac{\text { Right hand grip }- \text { Left hand grip }}{\text { Right hand grip }+ \text { Left hand grip }} * 100$,

where positive values indicate a right-hand superiority, and negative values indicate a leftward bias.

2) Pegboard LI $=\frac{\text { Left hand pegboard }- \text { Right hand pegboard }}{\text { Right hand pegboard }+ \text { Left hand pegboard }} * 100$, where positive values indicate a right-hand superiority, and negative values indicate a leftward bias (recall that pegboard scores are in seconds, so lower measures reflect better performance).

207 These LI's preserve directional bias within subjects in a way similar to the EHI—positive values 208 indicate rightward bias; negative values indicate leftward bias — and they also account for absolute 209 differences (i.e., magnitude differences in raw scores) between subjects (Brown et al., 2006; 210 Oldfield, 1971; but see McManus, Van Horn, and Bryden, 2016: 387-389). Discrepancies between 211 the EHI and these other handedness measures are the focus of the remaining sections. 


\begin{tabular}{|c|c|c|c|c|c|c|c|c|}
\hline \multicolumn{2}{|c|}{ Summary Statistics } & $\begin{array}{l}\text { Grip } \\
\text { strength } \\
\text { right hand } \\
\text { (pounds) }\end{array}$ & $\begin{array}{l}\text { Grip } \\
\text { strength } \\
\text { left hand } \\
\text { (pounds) }\end{array}$ & $\begin{array}{l}\text { Pegboard } \\
\text { right hand } \\
\text { (seconds) }\end{array}$ & $\begin{array}{l}\text { Pegboard } \\
\text { left hand } \\
\text { (seconds) }\end{array}$ & EHI & $\begin{array}{l}\text { Grip } \\
\text { strength } \\
\text { LI }\end{array}$ & $\begin{array}{l}\text { Pegboard } \\
\text { LI }\end{array}$ \\
\hline \multirow{4}{*}{$\begin{array}{l}\text { All } \\
\text { subjects } \\
n=1179\end{array}$} & mean & 86.531 & 81.167 & 21.630 & 22.604 & 66.068 & 3.426 & 2.270 \\
\hline & s.e. & 0.781 & 0.770 & 0.083 & 0.078 & 1.301 & 0.173 & 0.173 \\
\hline & variance & 26.804 & 26.443 & 2.839 & 2.667 & 1993.01 & 35.444 & 35.549 \\
\hline & st.dev. & 717.833 & 698.657 & 8.055 & 7.108 & 44.662 & 5.956 & 5.964 \\
\hline \multirow{4}{*}{$\begin{array}{l}\text { EHI+ } \\
n=1067\end{array}$} & mean & 86.242 & 80.403 & 21.521 & 22.686 & 78.932 & 3.780 & 2.699 \\
\hline & s.e. & 0.817 & 0.810 & 0.086 & 0.082 & 0.593 & 0.179 & 0.179 \\
\hline & variance & 26.686 & 26.472 & 2.821 & 2.681 & 374.913 & 34.295 & 34.321 \\
\hline & st.dev. & 711.475 & 700.086 & 7.953 & 7.179 & 19.372 & 5.859 & 5.861 \\
\hline \multirow{4}{*}{$\begin{array}{l}\text { EHI- } \\
n=112\end{array}$} & mean & 89.282 & 88.439 & 22.670 & 21.827 & -56.473 & 0.061 & -1.814 \\
\hline & s.e. & 2.634 & 2.376 & 0.266 & 0.228 & 2.710 & 0.552 & 0.510 \\
\hline & variance & 27.874 & 25.144 & 2.812 & 2.411 & 815.464 & 33.881 & 28.828 \\
\hline & st.dev. & 770.037 & 626.591 & 7.836 & 5.761 & 28.685 & 5.847 & 5.393 \\
\hline
\end{tabular}

Table 1: Summary statistics for the entire subject pool (top, $n=1179$ ), and the right-hander (middle) and left-hander

(bottom) subsets. Mean, standard error (s.e.), population variance, standard deviation (st.dev.) are shown for all groups for each of the following measures: raw Grip Strength (grip strength) scores for the right- and left-hands; raw Pegboard (pegboard) times (in seconds) for the right- and left-hands; EHI survey scores; and calculated Grip Strength and

\section{Results}

\section{Group-level differences in HCP handedness measures}

221 “j-shaped” skew which is present in EHI scores; grip strength and pegboard LI values are generally

222 centered around 0 and are more normally distributed, particularly in the case of EHI- subjects.

223 Although many of the HCP handedness measures are non-normally distributed, we use parametric

224 statistics in this study following the recommendation of Fagerland (2012), in which parametric

225 statistics were shown to be more appropriate for large-scale data sets, even in cases where the

226 distributions are skewed. All analyses were completed in R 3.5.1 (R core Team 2018). We used t-

227 tests to assess group-level differences in the raw NIH Toolbox measures, and all measures except

228 for right-hand grip strength showed significant differences between EHI+ and EHI- subjects (see

229 Table 2). 


\begin{tabular}{|l|l|r|r|r|r|r|}
\hline $\begin{array}{l}\boldsymbol{t} \text {-tests for EHI-based } \\
\text { handedness groups }\end{array}$ & $\begin{array}{l}\text { EHI+ } \\
\text { mean }\end{array}$ & $\begin{array}{l}\text { EHI- } \\
\text { mean }\end{array}$ & $\begin{array}{l}\text { Difference } \\
\text { in means }\end{array}$ & \multicolumn{1}{c|}{ t-value } & \multicolumn{1}{c|}{$\boldsymbol{p}$-value } & $\begin{array}{l}\text { Cohen's D } \\
\text { (effect size) }\end{array}$ \\
\hline grip strength right hand & 86.242 & 89.282 & -3.040 & 1.102 & 0.2723 & 0.113 \\
\hline grip strength left hand & 80.403 & 88.439 & -8.036 & 3.201 & $0.002 * *$ & 0.305 \\
\hline pegboard right hand & 21.521 & 22.670 & -1.149 & 4.175 & $<0.001 * * *$ & 0.419 \\
\hline pegboard left hand & 22.686 & 21.827 & 0.859 & -3.429 & $<0.001 * * *$ & 0.323 \\
\hline EHI & 78.932 & -56.473 & 135.405 & -48.80 & $<0.001 * * *$ & 6.627 \\
\hline Grip Strength LI & 3.780 & 0.061 & 3.719 & -6.403 & $<0.001 * * *$ & 0.635 \\
\hline Pegboard LI & 2.699 & -1.814 & 4.513 & -8.327 & $<0.001 * * *$ & 0.770 \\
\hline
\end{tabular}

Table 2: $t$-tests comparing right-handed subset (EHI+) means to left-handed subset (EHI-) means, for: raw grip strength for the right- and left-hands; raw pegboard times (in seconds) for the right- and left-hands; EHI values; and calculated grip strength and pegboard LI's. Means, difference in means, t-values, $\mathrm{p}$-values, and effect sizes are shown. $* p<0.05$

$* * p<0.01$

$* * * p<0.001$

It is often the case that significant differences in handedness measures within un-balanced samples are driven largely by the 'right-handed' participants (see Flindall \& Gonzalez, 2018: 8, strength difference in means $=9.75, t=6.451, p<0.001$, effect size $=0.385$; pegboard difference in means $=-2.54, t=-16.549, p<0.001$, effect size $=0.993)$, but EHI- subjects showed a significant difference only for the pegboard task (grip strength difference in means $=-6.203, t=$ 1.249, $p=0.216$, effect size $=0.291$; pegboard difference in means $=1.963, t=3.740, p<0.001$, effect size $=0.869)($ see Figure 1). As shown in Table 2, effect sizes between EHI+ and EHI-

249 between the three measures, indicating that increased rightward-bias in one measure correlates with increased rightward-bias in the others. Sub-sample tests, however, confirmed that this effect

251 was also driven by the EHI+ subjects, as only one of the EHI+ correlations reached significance,

252 and none of the EHI- correlations did (Table 3). Although we replicate group-level patterns 

scores may be poor representations of HCP subjects' actual manual performance, particularly for separate subsets (EHI+ and EHI-) in addition to running whole-group analyses.

\begin{tabular}{|l|r|r|r|r|r|r|}
\hline $\begin{array}{l}\text { Pearson's } \\
\text { correlations for } \\
\text { EHI groups }\end{array}$ & $\begin{array}{l}\text { EHI and grip } \\
\text { strength LI } \\
\text { correlation }(\boldsymbol{r})\end{array}$ & $\boldsymbol{p}$-value & $\begin{array}{l}\text { EHI and } \\
\text { pegboard LI } \\
\text { correlation }(\boldsymbol{r})\end{array}$ & $\boldsymbol{p}$-value & $\begin{array}{l}\text { Grip strength } \\
\text { and pegboard LI } \\
\text { correlation }(\boldsymbol{r})\end{array}$ & $\boldsymbol{p}$-value \\
\hline All subjects & 0.1866 & $<0.001 * * *$ & 0.2511 & $<0.001 * * *$ & 0.0848 & $0.003 * *$ \\
\hline EHI+ & 0.0443 & 0.15 & 0.1452 & $<0.001 * * *$ & 0.0515 & 0.09 \\
\hline EHI- & 0.1119 & 0.24 & -0.0406 & 0.67 & -0.0107 & 0.91 \\
\hline
\end{tabular}

Table 3: Pearson's linear correlation values for EHI and grip strength LI, EHI and pegboard LI, and grip strength and pegboard LI's. Correlation values ( $r$ ) and probabilities ( $p$ (uncorrelated)) are shown for all three tests for the entire sample (top), as well as the EHI+ subset (middle) and EHI- subset (bottom).

$* p<0.05$

$* * p<0.01$

$* * * p<0.001$

Raw Grip Strength and Pegboard Measures of Left and Right Hands For EHI- and EHI+ HCP Subjects, $n=1179$
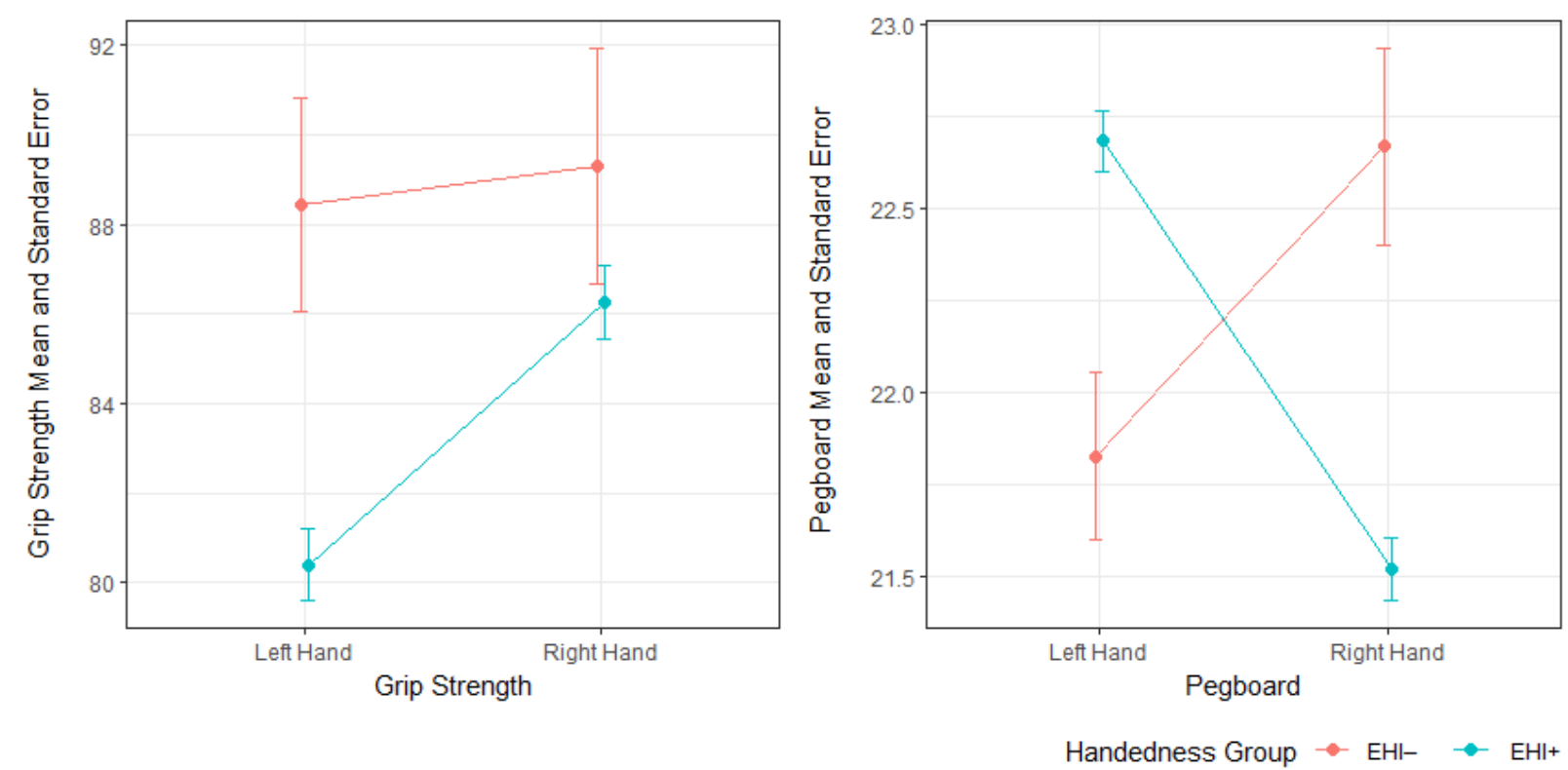

Figure 1: Interaction plots depicting raw grip strength (left) and raw pegboard (right) scores for EHI- (red) and EHI+ (aqua) subject subsets. Note that differences between the dominant and non-dominant hand are less severe in 
Characterizing handedness distributions: What about consistency?

That left- and right-handers show different patterns hand biases across multiple measures

272 is interesting on its own, but that these differences are unstable across multiple measures

273 complicates interpretations. An important discussion related to this about handedness consistency

274 (see Prichard, Propper, \& Christman, 2016), which is related to degree of preference. On this,

275 Leppanen and colleagues (2018) state:

"If handedness inventories are valid measures of preference, then reports of strong preference should be associated with relatively large performance disparities favoring the putatively preferred hand and reports of weak preference should be associated with relatively small disparities. To our knowledge, such a relationship has never been documented in the context of performing laboratory analogues of inventory tasks" (Leppanen et al., 2018: 544, emphasis added). task completion. Using a simple difference (non-dominant hand time - dominant hand time) for the timed version of each task, the team first noted that differences between hands were

290 each participant). As hand order was significant, the team did analyses for preferred-hand-first and

291 preferred-hand-second groups separately; they found significant correlations between EHI scores

292 and the magnitude of time difference between hands when performing survey tasks. Regarding 293 handedness consistency, Leppanen and colleagues split their data set into consistent (EHI +/- 80) 
294 and inconsistent (EHI between 0 and $+/-75$ ) handers, and found that proportion of tasks performed

295 with the preferred hand, and time differences between hands, were significantly higher in

296 consistent handers, with the odds of using the nonpreferred hand for spontaneous task performance

2978 times higher in inconsistent handers (Leppanen et al., 2018: 551).

298 We wanted to explore the HCP data in a similar way, so we split EHI+ and EHI299 participants by handedness consistency using the same EHI cutoff (scores $+/-80$ are considered 300 consistent handers, whereas inconsistent handers have EHI scores between 0 and $+/-75$ ). It is 301 important to note that a significantly higher proportion of EHI + participants are consistent handers $302(n=662,62.04 \%)$ when compared to EHI- participants $(n=30,26.78 \%$ classed as consistent; $\boldsymbol{\chi}-$ 303 squared for EHI and consistency $=50.527, p<0.001$ ), again likely reflecting left-hander's overall 304 tendency towards reduced manual bias. The only measure which showed significant differences 305 between consistent and inconsistent handers was the pegboard LI, and this was only in EHI+ 306 participants (difference of means $=1.3803, t=3.7157, p<0.001$, effect size $=0.238$ ). Unlike 307 Leppanen and colleagues (2018), and at a much larger sample size, we find that consistently308 handed HCP participants have similar distributions of all other behavioral handedness measures, 309 regardless of whether they are left-handed or right-handed (Figure 2). 


\section{Raw Behavioral Handedness Measures of Consistent and Inconsistent Handers}
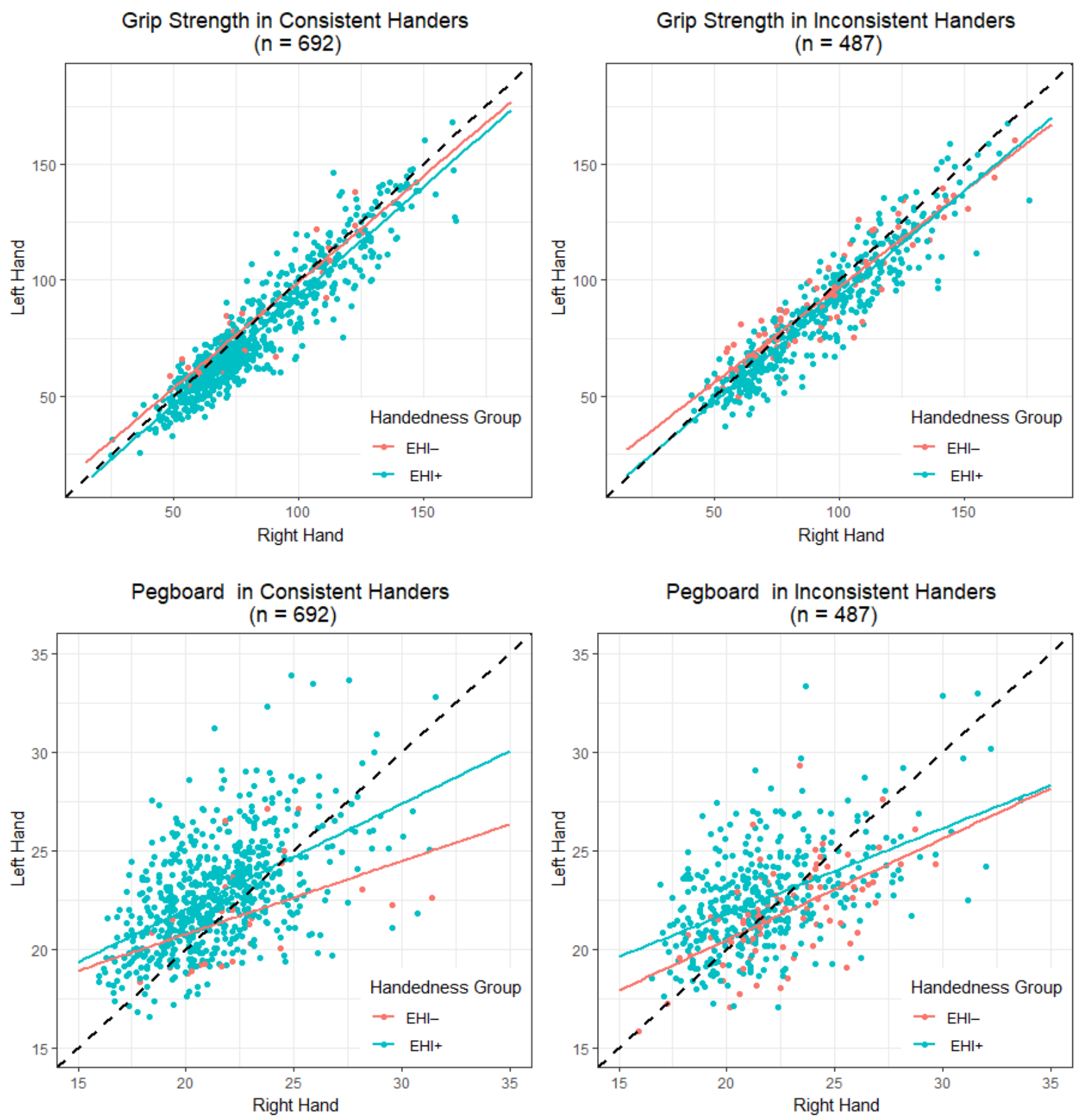

Figure 2: Raw scores for grip strength and pegboard in consistent vs. inconsistent handers. With the exception of pegboard scores, consistent and inconsistent handers (based on EHI scores) have indistinguishable behavioral 

subjects into meaningful handedness groups, but when evaluated against the behavioral measures, these procedures look more tenuous, which is concerning considering their ubiquity. McManus, Van Horn, and Bryden (2016) discussing related issues with regard to handedness characterization, revisiting data from Tapley and Bryden's (1985) circle-marking task ( $n=1556$, with $n=161$ selfidentified as left-handed), and Van Horn's (1992) study, which includes Tapley-Bryden circlemarking data as well as Annett pegboard data (where various pegboard configurations were tested). Although this team was not specifically interested in validating the EHI survey against

323 behavioral measures, but instead in the overall characterization of handedness, they note "almost 324 perfect separation of self-reported right and left-handers" on the Tapley-Bryden circle-marking task, with "only $7(0.5 \%)$ right-handers performing better with the left hand, and $1(0.6 \%)$ lefthanders performing better with the right hand" (McManus, Van Horn, \& Bryden, 2016: 378). McManus and colleagues (2016) found that direction of handedness across tasks was more consistent than degree of handedness, and note:

"If a person is right-handed for task $A$ then they are very likely to be right-handed for task then that has no predictive ability for how much better their dominant hand will be compared with their non-dominant hand on task B." (McManus, Van Horn, \& Bryden, 2016: 
subject-specific basis using the following categories: full congruency, where all three scores indicate the same directional bias (all positive, or all negative); partial congruency, where one of the behavioral LI's is in the opposite direction as the EHI; and non-congruency, where both the grip strength LI and pegboard LI indicate hand bias opposite to the EHI score. Congruency was assessed for the whole sample as well as the EHI+ and EHI- subsets (Table 4).

\begin{tabular}{|l|r|r|r|r|r|r|r|}
\hline Congruence & \multicolumn{1}{|c|}{ Total } & Congruent & Partial & Incongruent & \% Congruent & \% Partial & \% Incongruent \\
\hline All subjects & 1179 & 592 & 469 & 118 & 50.25 & 39.81 & 10.02 \\
\hline EHI+ & 1067 & 555 & 413 & 99 & 52.01 & 38.71 & 9.28 \\
\hline EHI- & 112 & 37 & 56 & 19 & 33.04 & 50.00 & 16.96 \\
\hline
\end{tabular}

Table 4: Congruency of EHI direction with both Grip Strength (grip strength LI) and Pegboard (pegboard LI) for all subjects (top), the EHI+ subset (middle), and the EHI- subset (bottom). Congruency was assessed for each subject, where positive values indicate right-hand skew and negative values indicate left-hand skew for all three measures. Frequencies for congruent subjects (all measures match), partial congruence (EHI matches either grip strength LI or pegboard LI), and non-congruent (EHI does not match either grip strength LI or pegboard LI), are shown in the left, and percentages on the right. Note that roughly half of the overall sample has at least one behavioral score indicating opposing hand preference to the EHI, and more than $15 \%$ of left-handers (EHI-) have behavioral scores which both indicate right-hand preference. have behavioral LI's which both indicate hand performance opposite to their EHI scores, regardless of whether they report as right-handed or left-handed. Intra-class correlations (ICC's) for EHI, grip strength LI, and pegboard LI directionality (Koo et al., 2016) show low

359 correspondence $(<0.2$, indicating only slight agreement $)$ for the whole sample, and for the EHI+ and EHI - subsets as well, confirming our other analyses (see Supplementary Materials, Table 1).

361 Interestingly, congruence frequencies are similar when the data are further split by handedness 


\section{Handedness Measure Congruency between EHI, GSLI, and PBLI}

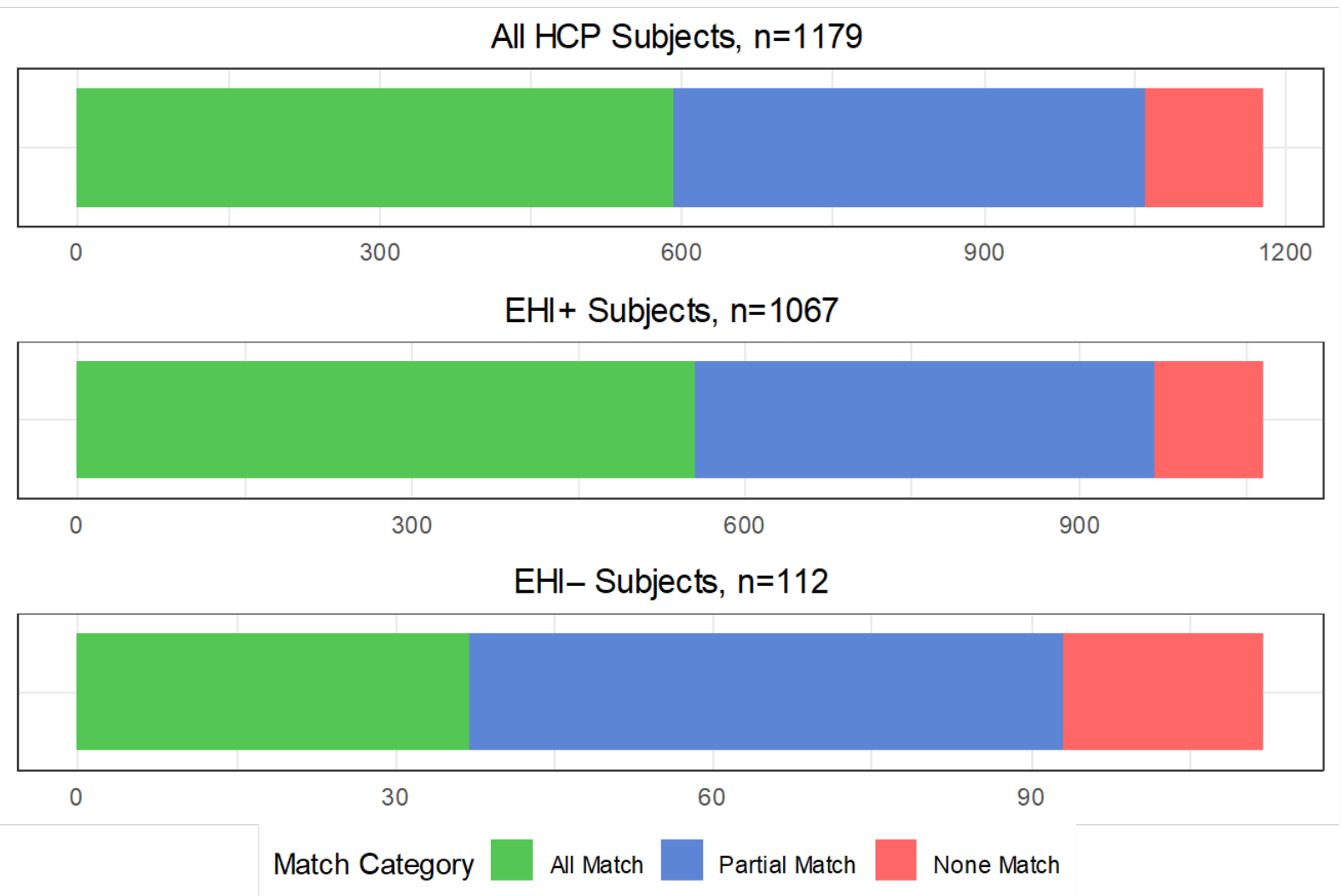

Figure 3: Congruency plots for the entire sample (left), EHI- (center) and EHI+ (right) subjects. A minority of EHIsubjects have all three scores matching in direction, and roughly half of all subjects have either grip strength LI or pegboard LI scores indicating hand performance bias opposite in direction to their EHI scores.

Discussing the Van Horn (1988) data, McManus and colleagues (2016) note that pegboard

370 data are unimodal, as opposed to both the circle marking task (which is bimodal) and survey data

371 (which is " $\mathrm{j}$-shaped"). On this, they claim that "the T\&B [circle] task separates the right- and left-

372 handers entirely, whereas the pegboard scores are not so good at doing that, four of the 28 right-

373 handers and 2 of the 28 left-handers being in the "wrong" half" (McManus, Van Horn, \& Bryden,

374 2016: 384, emphasis added). We have found very different proportions of congruency in the HCP

375 data, although, if anything, this confirms the authors original point that different handedness

376 measures simply behave differently. 
Unfortunately, knowing all the ways in which multiple handedness measures do not correspond with each other does very little to tell researchers which measure might be the best one to use in analyses about handedness. Of potential relevance here is the HCP's test-retest data ${ }^{2}$, in which EHI, grip strength, and pegboard measures were collected on two separate occasions for 45 participants. Test-retest reliability was computed using Pearson's correlations between initial test scores and retest scores (Table 5). Regarding self-report and survey-based EHI data, we replicate Flindall and Gonzalez's (2018) findings that survey scores are generally stable across testing sessions, with the exception of the Broom question ("Which hand would you use to hold a broom (upper hand)?”), and Eye question (“Which eye do you use when using only one?”). Raw grip

387 strength measures have high reliability as well, although raw pegboard scores have only moderate 388 reliability. Presumably, measures with higher test-retest reliability should be favored, as low 389 reliability likely indicates high measurement error. Despite high and moderate reliability at the raw 390 score level, reliability scores for grip strength and pegboard laterality indices (or for raw 391 differences; see McManus, Van Horn, \& Bryden, 2016: 387-388) are much lower. It is unclear to 392 us why this might be, but overall, it seems that as one looks at handedness data for these large393 scale data sets more thoroughly, there is less and less clarity about how they should best be used.

\footnotetext{
${ }^{2}$ We had to ask for the raw NIH toolbox scores for the test-retest data, which lead us to an interesting discovery about the HCP's definition of "raw" NIH toolbox data. It seems that the HCP "raw" NIH toolbox data for the 45 test-retest participants in the larger s1200 data release is actually an average of the two scores from T1 and T2, meaning that for these participants, our laterality indices (Grip strength LI and Pegboard LI) were computed on averaged "raw" data, unbeknownst to us, when the original analyses took place, whereas the remaining 1134 participant LI's were calculated on single-measure (truly "raw") data.
} 


\begin{tabular}{|l|r|r|}
\hline $\begin{array}{l}\text { Pearson's linear correlations } \\
\text { for test-retest reliability }\end{array}$ & Correlation $(\boldsymbol{r})$ & \multicolumn{1}{l|}{ p-value } \\
\hline Self-identification & 1 & 0 \\
\hline EHI & 0.975 & $<0.001^{* * *}$ \\
\hline Write & 0.993 & $<0.001^{* * *}$ \\
\hline Throw & 0.966 & $<0.001^{* * *}$ \\
\hline Scissors & 0.947 & $<0.001^{* * *}$ \\
\hline Toothbrush & 0.862 & $<0.001^{* * *}$ \\
\hline Knife & 0.734 & $<0.001^{* * *}$ \\
\hline Spoon & 0.878 & $<0.001^{* * *}$ \\
\hline Broom & 0.620 & 0.0031 \\
\hline Match & 0.894 & $<0.001^{* * *}$ \\
\hline Box & 0.792 & $<0.001^{* * *}$ \\
\hline Foot & 0.794 & $<0.001^{* * *}$ \\
\hline Eye & 0.505 & 0.2227 \\
\hline Grip strength right hand & 0.907 & $<0.001^{* * *}$ \\
\hline Grip strength left hand & 0.939 & $<0.001^{* * *}$ \\
\hline Pegboard right hand & 0.525 & $<0.001^{* * *}$ \\
\hline Pegboard left hand & 0.556 & $<0.001^{* * *}$ \\
\hline Grip strength LI & 0.417 & $0.0042^{* *}$ \\
\hline Pegboard LI & 0.277 & 0.0646 \\
\hline Grip strength difference & 0.408 & $0.0053^{*}$ \\
\hline Pegboard difference & $0.0462^{*}$ \\
\hline Tab & 0.298 & \\
\hline
\end{tabular}

394 Table 5: Test-retest reliability measures, using Pearson's linear correlations, for the entire test-retest sample $(n=45)$.

395 Correlation values $(r)$ and probabilities ( $p$ (uncorrelated)) are shown for self-identification, overall EHI scores, the 396 individual survey question answers, raw grip strength and pegboard scores, and laterality indices and raw differences 397 between these measures for the right and left hands.

$398 * p<0.05$

$399 * * * p<0.01$

$400 \quad * * * p<0.001$

401

\section{A multivariate approach for aggregating handedness scores}

404 we are ultimately less interested in predicting handedness from one measure to the next, and are

405 instead more concerned with implications for subject classification and exclusion in neuroimaging

406 contexts. Knowing that roughly half of the HCP subjects have partially or entirely mismatched

407 survey-based and behavior-based handedness scores, and that each of these measures is genuinely

408 complex in terms of its reliability and potential external validity, what procedures should be used

409 to split subjects into groups for analysis? What, if anything, should be done about the use of the 
410 EHI for subject recruitment and screening? Results of many studies could change if subjects were

411 grouped as 'right-' or 'left-handed' based not on EHI scores, but on Grip Strength (grip strength

$412 \mathrm{LI}+n=845$ (71.7\%); grip strength LI $-n=334(29.3 \%)$ ), Pegboard (pegboard LI $+n=774$

413 (65.6\%); pegboard LI- $n=405(35.4 \%))$, or other handedness measures. Unfortunately, so little

414 has been done to assess these measures in their own right that much more needs to be done to

415 assess their validity if we are to justify their use in place of the EHI. In lieu of this, we advocate

416 for a multivariate approach (following Corey et al., 2001) by applying a Principal Component

417 Analysis to the EHI, grip strength LI and pegboard LI scores together. Principal Component

418 Analysis (PCA) is a widely used statistical approach for assessing the degree to which multiple

419 variables can (or cannot) be reduced to a smaller number of dimensions (Wold, Esbensen, \&

420 Geladi, 1987). PCA analysis including the EHI, grip strength LI, and pegboard LI, resulted in three

421 components (PC's, see Table 6): PC1 (45.19\% variance explained) has positive loadings on all

422 three handedness measures, and we suggest it as a good measure of overall handedness (with

423 negative scores reflecting overall left-hand bias, positive values reflecting overall right-hand bias),

424 and intermediate values reflecting inconsistent or mixed handedness (Figure 4). PC2 (30.67\%

425 variance explained) appears to represent a dexterity vs. strength tradeoff (positive grip strength LI

426 loading, negative pegboard LI loading), and PC3 (24.14\% variance explained) contrasts the EHI

427 (self-reported) against the behavioral handedness measures.

\begin{tabular}{|l|r|r|r|}
\hline PCA Loadings & \multicolumn{1}{|l|}{ PC1 } & \multicolumn{1}{l|}{ PC2 } & \multicolumn{1}{l|}{ PC3 } \\
\hline EHI & 0.659 & -0.088 & 0.747 \\
\hline Grip strength LI & 0.577 & 0.812 & -0.330 \\
\hline Pegboard LI & 0.482 & -0.578 & -0.577 \\
\hline
\end{tabular}

428 Table 6: Loadings for EHI, grip strength LI, and pegboard LI on PC1, PC2, and PC3. 


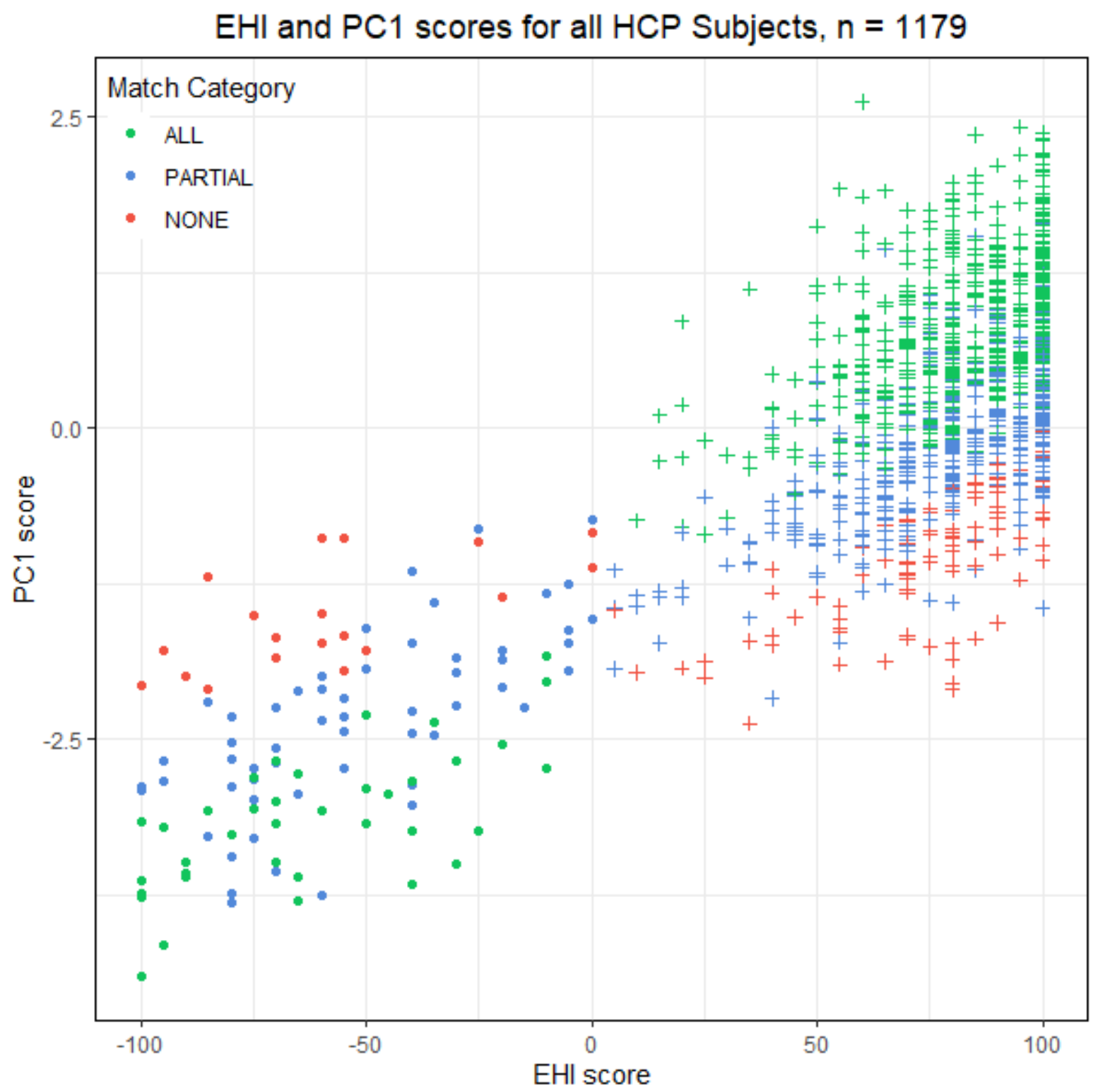

Figure 4: EHI and PC1 scores showing congruency categories for all HCP participants. EHI- participants are circles, EHI+ participants are crosses. PC1 scores generally track with EHI scores, but they also incorporate congruence data, as many EHI+ participants with partial or non-matched behavioral scores (i.e., left-hand-biased grip strength and/or pegboard LI's) have negative PC1 values.

We will focus the rest of our discussion primarily on PC1 as an overall handedness measure. As PC1 encompasses all three handedness measures, we explored the effects of using PC1 scores, not the EHI or other raw handedness measures, as our variable for grouping participants. We repeated many of the analyses conducted on EHI+ and EHI- subjects, instead on $\mathrm{PC} 1+(n=510)$ and PC1- $(n=669)$ subjects. T-tests on the raw behavioral scores show significant 
441 from the EHI-based analyses (Table 7; compare to Table 2). As PC1 is a reformulation of the EHI

442 and behavioral LI's, each of these measures also differs significantly between PC1+ and PC1-

443 participants (Figure 5). As discussed in our section on handedness consistency, a higher percentage

444 of EHI+ participants were considered consistent handers compared to EHI- participants. When

445 split by PC1 scores rather than EHI scores, handedness consistency information is still salient $(\chi-$

446 squared for EHI and consistency $=125.51, p<0.001)$, with significantly more consistent handers

447 in the PC1+ group ( $n=487,72.8 \%)$, and significantly less consistent handers in the PC $1-$ group

$448 \quad(n=205,40.2 \%)$. These results provide support for grouping subjects based on PC1, and further

449 suggest that it might be a more encompassing, and thus more appropriate characterization of

450 handedness in HCP participants.

\begin{tabular}{|l|r|r|r|r|r|r|}
\hline $\begin{array}{l}\boldsymbol{t} \text {-tests for PC1-based } \\
\text { handedness groups }\end{array}$ & $\begin{array}{l}\text { PC1+ } \\
\text { mean }\end{array}$ & $\begin{array}{l}\text { PC1- } \\
\text { mean }\end{array}$ & $\begin{array}{l}\text { Difference } \\
\text { in Means }\end{array}$ & \multicolumn{1}{c|}{-value } & \multicolumn{1}{|c|}{$\boldsymbol{p}$-value } & $\begin{array}{l}\text { Cohen's D } \\
\text { (effect size) }\end{array}$ \\
\hline grip strength right hand & 84.007 & 89.841 & -5.834 & 3.672 & $<0.001 * * *$ & 0.219 \\
\hline grip strength left hand & 75.022 & 89.227 & -14.205 & 9.308 & $<0.001 * * *$ & 0.557 \\
\hline pegboard right hand & 21.521 & 22.778 & -1.257 & 12.975 & $<0.001 * * *$ & 0.785 \\
\hline pegboard left hand & 22.686 & 21.913 & 0.773 & -8.056 & $<0.001 * * *$ & 0.467 \\
\hline EHI & 84.417 & 42.000 & 42.417 & -16.268 & $<0.001 * * *$ & 1.076 \\
\hline Grip Strength LI & 5.885 & 0.202 & 5.683 & -25.725 & $<0.001^{* * *}$ & 1.082 \\
\hline Pegboard LI & 5.398 & -1.832 & 7.230 & -18.673 & $<0.001 * * *$ & 1.516 \\
\hline
\end{tabular}

Table 7: $t$-tests comparing PC1+ to PC1- means, for: raw grip strength for the right- and left-hands; raw pegboard 452 times (in seconds) for the right- and left-hands; EHI values; and calculated grip strength and pegboard LI's. Means, 
bioRxiv preprint doi: https://doi.org/10.1101/2020.03.08.982678; this version posted March 9, 2020. The copyright holder for this preprint (which was not certified by peer review) is the author/funder. All rights reserved. No reuse allowed without permission.

\section{PC1 and EHI comparisons for Behavioral LI's for all HCP Subjects, $n=1179$}
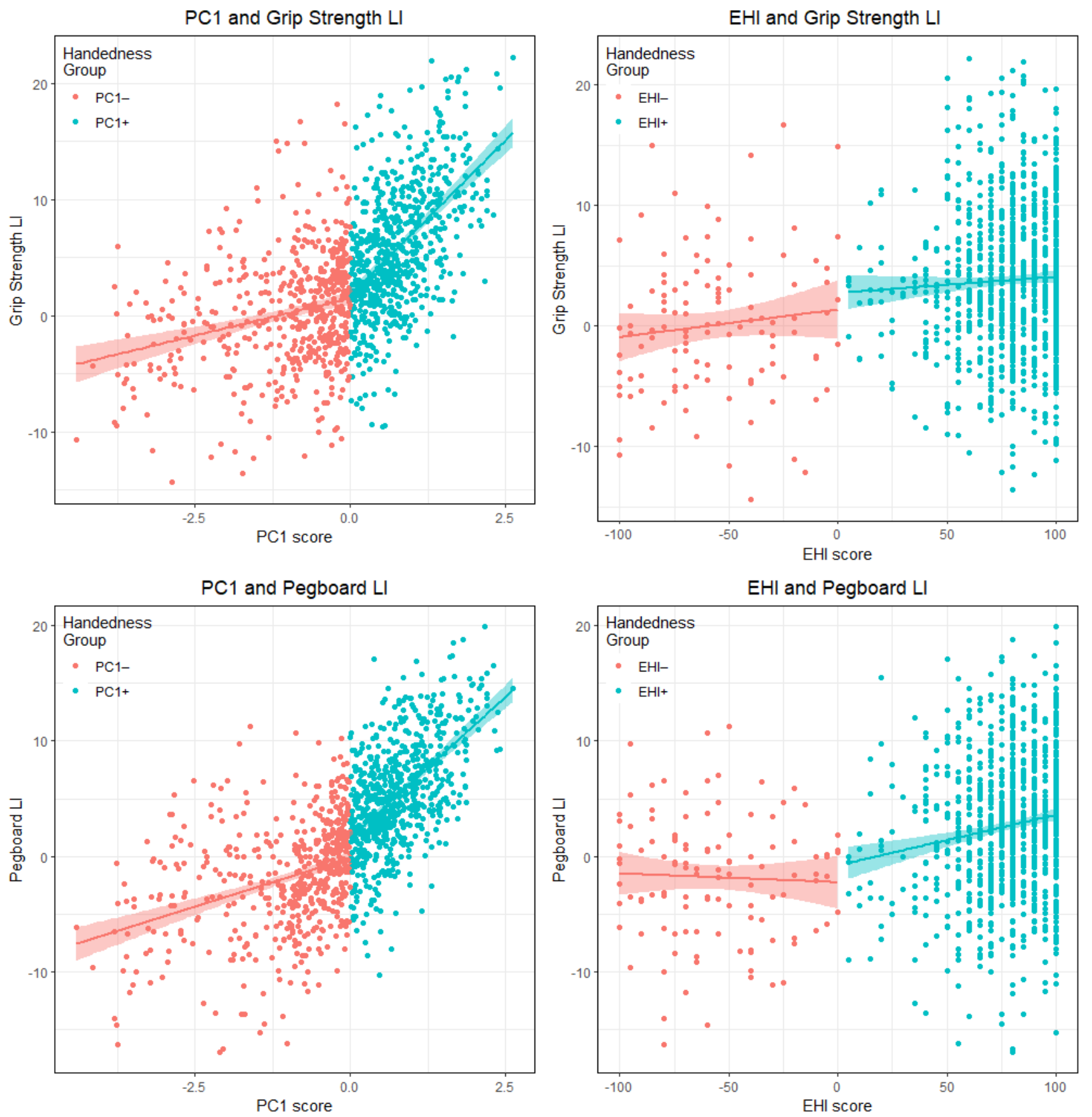

Figure 5: Comparison of behavioral handedness LI's between PC1-based (left) and EHI-based (right) partitions of 
In our final analysis, we explore how one might use PCA scores to address subject-specific disparities in their own data, in cases where multiple assessments of hand preference or

464 performance were collected and a PCA can be run. As mentioned earlier, the HCP is a large-sample open neuroimaging initiative, so there is much that could be done in the future to explore how different handedness classification methods effect analyses on psychometric, anatomical, and functional brain data. Here, we use FreeSurfer brain segmentation data, available for direct download via the HCP, to illustrate the concept. Of the 1179 subjects with handedness data, 1092 $(\mathrm{EHI}+n=991, \mathrm{EHI}-n=101 ; \mathrm{PC} 1+n=510, \mathrm{PC} 1-n=669)$ have cortical and subcortical segmentation data from the HCP FreeSurfer pipeline, which categorizes each voxel into one of over 30 cortical and subcortical classes (Fischl et al., 2002). FreeSurfer segmentations for each

472 subject come from the HCP as whole-brain volumes (for example: white matter volume, sub-

473 cortical grey matter volume, etc.), and as region-based surface areas, thicknesses, and volumes for 474 the left- and right-hemispheres separately (for example, left-hemisphere pars opercularis area, 475 right-hemisphere pars triangularis volume, etc.). In cases where paired left- and right-hemisphere 476 data were given for a region, we calculated a laterality index (LI) for that region as before with the 477 handedness measures, using the following formula:

3) FreeSurfer regional LI $=\frac{\text { Left hemisphere measure-Right hemisphere measure }}{\text { Left hemisphere measure+Right hemisphere measure }} * 100$, where positive values indicate a larger left-hemisphere measure, and negative values indicate a larger right-hemisphere measure.

481 This resulted in over 100 brain-based variables: 17 whole-brain volumes, volumetric LI's for 16 482 regions, 34 surface area LI's and 34 cortical thickness LI's (see Supplementary Materials, Table 2 483 for a full list of Freesurfer regions). 
Similar tests were run on the Freesurfer data (measured microliters $(\mu 1)$ ) in as on the handedness metrics, although we applied Bonferroni corrections to these tests, as there were over 100 Freesurfer variables to test. T-tests were run on Freesurfer variables using subject groupings based on both the EHI and PC1 scores. For a majority of the Freesurfer measures, no significant differences were found between subject groups, regardless of whether they were defined by EHI scores or PC1 scores. However, many of the whole-brain volume measures were significantly different between PC1- and PC1+ subjects. After Bonferroni correction, Freesurfer data for the

491 following regions showed significant differences between $\mathrm{PC} 1+$ and $\mathrm{PC} 1-$ participants:

492 Intracranial volume, Brain Segmentation volume, Cortical Grey Matter volume, Total Grey Matter

493 volume, Total White Matter volume, and Supratentorial volume. Only Lateral Orbitofrontal area

494 LI showed significant differences between EHI+ and EHI- participants, with all other comparisons

495 resulting in nonsignificant corrected p-values (Table 8). Overall, we believe this provides tentative

496 support for the use of PC1 scores as at least as good a dependent variable for subject handedness

497 in future analyses as the EHI, although much more can be done to validate our approach with the

498 other HCP neuroimaging data in the future. As many of these are raw measures, not LI's, they do

499 not directly relate to brain lateralization, but instead show that PC1- subjects in this sample have

500 larger brains overall than PC1+ subjects (at $\alpha=0.05)$. Linear models were run to explore the effects

501 of age and sex on the Freesurfer findings, and in all cases, sex was the only significant predictor

502 variable. Although there are similar proportions of males and females for the EHI-based grouping

$503(\chi$-squared for EHI and sex $=2.612, p=0.106)$, there are more females in the HCP sample overall

$504(\mathrm{~F}=638,55 \%)$, and a significantly higher proportion of $\mathrm{PC} 1+$ females when the data are

505 partitioned using PC1 scores $(\chi$-squared for PC1 and sex $=32.707, p<0.001)$. Interpretations for

506 the Freesurfer data depend on further exploration of the relationship between handedness and sex

507 in the HCP data, a fruitful avenue for future research. 


\begin{tabular}{|l|r|r|r|r|}
\hline Whole Brain volumes & PC1 t-value & $\boldsymbol{p}$-value & EHI t-value & $\boldsymbol{p}$-value \\
\hline Intracranial volume & -3.923 & $\mathbf{0 . 0 0 9} * *$ & -0.095 & NS \\
\hline Brain Segmentation volume & -4.162 & $\mathbf{0 . 0 0 3} * *$ & -0.363 & NS \\
\hline Cortical Grey Matter volume & -4.102 & $\mathbf{0 . 0 0 4} * *$ & -0.285 & NS \\
\hline Total Grey Matter volume & -4.391 & $\mathbf{0 . 0 0 1} * * *$ & -0.331 & NS \\
\hline Total White Matter volume & -3.466 & $\mathbf{0 . 0 5 0} *$ & -0.210 & NS \\
\hline Supratentorial volume & -3.974 & $\mathbf{0 . 0 0 8} * *$ & -0.334 & NS \\
\hline Surface area LI's & $\mathbf{t}$ & $\mathbf{p}$-value & t & p-value \\
\hline Lateral Orbitofrontal area LI & -0.423 & NS & 2.049 & $\mathbf{0 . 0 4 3}$ \\
\hline
\end{tabular}

Table 8: T-tests for Freesurfer data in PC1-based (left) and EHI-based (right) HCP data partitions. Only areas with significant Bonferroni-corrected $p$-values are shown (see Supplemental Data table 2 for a full list of Freesurfer regions). Bolded rows are significant at $\alpha=0.05$, and NS denotes non-significant $p$-values.

$* p<0.05$

$* * p<0.01$

$* * * p<0.001$

\section{Discussion}

In many ways, our results mirror previous investigations into the EHI as a measure of subject handedness in many aspects, but there are a few important differences. With a much larger

519 EHI as a sole handedness metric. Based on our sub-sample analyses using the EHI to split subjects

520 into groups, it seems that right- and left-handers have very different manifestations of multiple

521 handedness measures. Future works on handedness-related asymmetries would benefit from

522 whole-group as well as sub-sample reporting, as whole-sample analyses may be 'benefitting' from

523 these disparities. For example, although the raw measures (grip strength and pegboard scores for

524 the right and left hands) and LI's had significant pair-wise correlations for the whole sample, our

525 results corroborate other works in showing low correspondence in particular between EHI and grip

526 strength LI, and we have also shown that — when considered separately-EHI, pegboard LI, and

527 grip strength LI scores for EHI- subjects do not correlate with each other hardly at all. Although

528 this not the case in some recent studies, many published works present analyses of their data for

529 all subjects as one group, leaving us unsure of how much their effects are being driven by EHI+

530 subjects (especially in studies using non-balanced samples, such as our own). 
The EHI no doubt communicates some credible information about subject handedness, but

532 we have also shown that at least $10 \%$ of the entire HCP sample shows non-congruence between

533 the survey- and behavioral-measures for handedness. Alarmingly, over $65 \%$ of EHI- subjects

534 have at least one directional disparity between EHI scores and Grip Strength and Pegboard LI's.

535 For those uninterested in studying handedness (those who purposely exclude 'left-handers' from

536 their studies), these congruence results may also matter, as the most widely used criterion for left-

537 hand subject exclusion in neuroimaging projects - a negative EHI score - is frequently not

538 congruent with actual behavioral data, at least in the HCP subject pool. Regarding the pragmatics

539 of screening for right-hand-exclusive paradigms, however, we believe the continued use of the

540 EHI to be an appropriate course of action, particularly for participants with extreme EHI scores.

541 However, for those explicitly studying handedness, our congruence data urge extreme caution for

542 categorizing subjects based on EHI scores, particularly those with mid-range EHI values, and

543 especially in studies specifically on handedness.

544 It is still generally unclear which handedness measures should be trusted more over the

545 others in situations where they do not correlate, likely contributing to the continued use of only

546 one measure, typically a survey-based one such as the EHI, in neuroimaging contexts. We have

547 proposed an alternative approach for those who have previously collected multiple measures of

548 handedness, which is to aggregate subject handedness data via Principal Components Analysis and

549 use PC1 as an overall handedness metric (instead of EHI scores) as the independent variable for

550 analyses. The benefit of this approach is that any number of handedness measures can be added to

551 an analysis of this kind, which in turn may allow us to evaluate one measure against another in

552 future studies. We have shown that many features of EHI-based analyses (correlations across

553 measures, handedness consistency, etc.) are preserved when using PC1-based analyses, suggesting

554 at the very least that this technique should be applied to additional data sets. A large caveat for this 
approach, however, is that data sets with different handedness measures may lead to very different

556 PCA results and thus different PC1 values, than our specific example here. For example, if finger

557 tapping were used instead of pegboard, it is not clear whether any of the resulting components

558 would have similar loadings to our own, and so there might be a lack of an overall measure of

559 handedness, as we have showed PC1 to be. The impact of different handedness measures on

560 resulting PC's should be further explored in future work on data sets both large and small.

In cases where EHI scores were the only handedness information collected, researchers can

562 still contribute improve upon the discourse on handedness-related asymmetries by "reining in"

563 their titles and text; instead of claiming that 'Handedness does or does not correlate with X' we

564 might be more direct: 'EHI scores do or do not correlate with X.' After decades of conflicting

565 results, it is time to seriously consider the value of the EHI as a sole proxy for subject handedness

566 in lateralization studies. As Oldfield himself stated: "I am far from suggesting that, where manual

567 or cerebral laterality are important issues in a piece of research, the [EHI] is a sufficient means of

568 assessment of the handedness aspect. But for screening purposes...it may, I hope, prove useful”

569 (1971: 110). We feel that researchers interested in laterality need to acknowledge that many of our

570 problems in identifying handedness-related and handedness-independent asymmetries may be

571 related largely to methods which are confounding, not clarifying, potentially real relationships.

572 The best way to address this issue is to replicate works like those of Gonzalez and colleagues and

573 use more naturalistic measures of handedness, even if they are labor-intensive. In lieu of this, and

574 to make use of extant data such as that provided by the HCP and other open-source neuroimaging

575 initiatives, it will also be important to compare each handedness measure to well-known

576 asymmetries in the brain, perhaps starting with fMRI of language tasks, but extending beyond that.

577 Careful evaluation of these measures may confirm the EHI as an important handedness measure

578 to include in data analysis, but there is no sure way to know this without explicitly testing it. 


\section{Acknowledgements}

This research was supported in part by grant 52935 from the John Templeton Foundation titled:

"What Drives Human Cognitive Evolution?"

\section{Disclosure of interest}

The authors report no conflict of interest.

\section{Data availability statement}

The data which support the findings of this study are available from the Human Connectome Project (HCP). Some restrictions apply to the availability of these data, which were used under approval of the HCP for this study. Supporting data and R code for PCA calculations (for data like our own) can be made available from the corresponding author, LMR, under reasonable request. However, raw handedness measures, congruency data, and FreeSurfer values can only be provided when requests are accompanied by proof of prior permission for restricted data usage directly from the HCP (as detailed at https://www.humanconnectome.org/study/hcp-young-adult/document/ restricted-data-usage). 


\section{References}

Annett, M. (1985). Which theory fails? A reply to McManus. British Journal of Psychology, 76(1), 17-29. https://doi.org/10.1111/j.2044-8295.1985.tb01927.x

Badzakova-Trajkov, G, Corballis, MC, \& Häberling, IS. (2016). Complementarity or independence of hemispheric specializations? A brief review. Neuropsychologia, 93, 386393. https://doi.org/10.1016/J.NEUROPSYCHOLOGIA.2015.12.018

Bailey, L. M., McMillan, L. E., \& Newman, A. J. (2019). A sinister subject: Quantifying handedness-based recruitment biases in current neuroimaging research. European Journal of Neuroscience, ejn.14542. https://doi.org/10.1111/ejn.14542

Barch, D. M., Burgess, G. C., Harms, M. P., Petersen, S. E., Schlaggar, B. L., Corbetta, M., ... Van Essen, D. C. (2013). Function in the human connectome: Task-fMRI and individual differences in behavior. NeuroImage, 80, 1112-189. https://doi.org/10.1016/j.neuroimage.2013.05.033

Brown, S. G., Roy, E. A., Rohr, L. E., \& Bryden, P. J. (2006). Using hand performance measures to predict handedness. Laterality: Asymmetries of Body, Brain and Cognition. https://doi.org/10.1080/1357650054200000440

Brown, S. G., Roy, E. A., Rohr, L. E., Snider, B. R., \& Bryden, P. J. (2004). Preference and performance measures of handedness. Brain and Cognition, 55(2), 283-285. https://doi.org/10.1016/J.BANDC.2004.02.010

Bryden, M. P. (1977). Measuring handedness with questionnaires. Neuropsychologia, 15(4-5), 617-624. https://doi.org/10.1016/0028-3932(77)90067-7

Bryden, P. J., Brown, S. G., \& Roy, E. A. (2011). Can an observational method of assessing hand preference be used to predict language lateralisation? Laterality: Asymmetries of Body, Brain and Cognition. https://doi.org/10.1080/1357650X.2010.513386

Büsch, D., Hagemann, N., \& Bender, N. (2010). The dimensionality of the Edinburgh Handedness Inventory: An analysis with models of the item response theory. Laterality: Asymmetries of Body, Brain and Cognition, 15(6), 610-628. https://doi.org/10.1080/13576500903081806

Christman, S. D., Prichard, E. C., \& Corser, R. (2015). Factor analysis of the Edinburgh Handedness Inventory: Inconsistent handedness yields a two-factor solution. Brain and Cognition, 98, 82-86. https://doi.org/10.1016/J.BANDC.2015.06.005

Corey, D. M., Hurley, M. M., \& Foundas, A. L. (). Right and left handedness defined: a multivariate approach using hand preference and hand performance measures. Neuropsychiatry, Neuropsychology, and Behavioral Neurology, 14(3), 144-52. Retrieved from http://www.ncbi.nlm.nih.gov/pubmed/11513097

Dragovic, M. (2004). Towards an improved measure of the Edinburgh Handedness Inventory: A one-factor congeneric measurement model using confirmatory factor analysis. Laterality: Asymmetries of Body, Brain and Cognition, 9(4), 411-419. https://doi.org/10.1080/13576500342000248

Edlin, J. M., Leppanen, M. L., Fain, R. J., Hackländer, R. P., Hanaver-Torrez, S. D., \& Lyle, K. B. (2015). On the use (and misuse?) of the Edinburgh Handedness Inventory. Brain and Cognition, 94, 44-51. https://doi.org/10.1016/J.BANDC.2015.01.003

Fagerland, M. W. (2012). t-tests, non-parametric tests, and large studies-a paradox of statistical practice? BMC Medical Research Methodology 12, 78. https://doi.org/10.1186/1471-2288$12-78$ 
Fazio, R. L., \& Cantor, J. M. (2015). Factor Structure of the Edinburgh Handedness Inventory Versus the Fazio Laterality Inventory in a Population With Established Atypical Handedness. Applied Neuropsychology: Adult, 22(2), 156-160. https://doi.org/10.1080/23279095.2014.940043

Fazio, R., Coenen, C., \& Denney, R. L. (2012). The original instructions for the Edinburgh Handedness Inventory are misunderstood by a majority of participants. Laterality: Asymmetries of Body, Brain and Cognition, 17(1), 70-77. https://doi.org/10.1080/1357650X.2010.532801

Fazio, R., Dunham, K. J., Griswold, S., \& Denney, R. L. (2013). An Improved Measure of Handedness: The Fazio Laterality Inventory. Applied Neuropsychology, 1-6. https://doi.org/10.1080/09084282.2012.684115

Fischl, B., Salat, D. H., Busa, E., Albert, M., Dieterich, M., Haselgrove, C., van der Kouwe, A., Killiany, R., Kennedy, D., Klaveness, S., Montillo, A., Makris, N., Rosen, B., Dale, A. M., (2002). Whole brain segmentation: automated labeling of neuroanatomical structures in the human brain. Neuron 33, 341-355. http://www.ncbi.nlm.nih.gov/pubmed/11832223

Flindall, J. W., \& Gonzalez, C. L. R. (2018). Wait wait, don't tell me: Handedness questionnaires do not predict hand preference for grasping. Laterality: Asymmetries of Body, Brain and Cognition, 1-21. https://doi.org/10.1080/1357650X.2018.1494184

Gershon, R. C., Cella, D., Fox, N. A., Havlik, R. J., Hendrie, H. C., \& Wagster, M. V. (2010). Assessment of neurological and behavioural function: the NIH Toolbox. The Lancet Neurology, 9(2), 138-139. https://doi.org/10.1016/S1474-4422(09)70335-7

Gonzalez, C. L. R., \& Goodale, M. A. (2009). Hand preference for precision grasping predicts language lateralization. Neuropsychologia, 47(14), 3182-9. https://doi.org/10.1016/j.neuropsychologia.2009.07.019

Gonzalez, C. L.R., van Rootselaar, N. A., \& Gibb R. L. (2018). Sensorimotor lateralization scaffolds cognitive specialization. Progress in brain research 238: 405-433.

Gonzalez, C. L. R., Whitwell, R. L., Morrissey, B., Ganel, T., \& Goodale, M. A. (2007). Left handedness does not extend to visually guided precision grasping. Experimental Brain Research, 182(2), 275-9. https://doi.org/10.1007/s00221-007-1090-1

Hopkins, B. (2018). A review of performance asymmetries in hand skill in nonhuman primates with a special emphasis on chimpanzees. Progress in brain research 238: 57-90.

Kallen, M., Slotkin, J., Griffinth, J., Magasi, M., Salsman, J., Nowinski, C., \& Gershon, R. (2012a). NIH Toolbox 9-Hole Pegboard Dexterity Task. NIH Toolbox Technical Manual. Available from http://www.healthmeasures.net/images/nihtoolbox/Technical_Manuals/ Motor/Toolbox 9-Hole Pegboard Dexterity Test Technical Manual.pdf

Kallen, M., Slotkin, J., Griffinth, J., Magasi, M., Salsman, J., Nowinski, C., \& Gershon, R. (2012b). NIH Toolbox Grip Strength Test. NIH Toolbox Technical Manual. Available from http://www.healthmeasures.net/images/nihtoolbox/Technical_Manuals/Motor/ Toolbox_Grip_Strength_Test_Technical_Manual.pdf

Kaploun, K. A., \& Abeare, C. A. (2010). A comparison of four handedness classification schemes through the investigation of lateralised semantic priming. Laterality: Asymmetries of Body, Brain and Cognition, 15(5), 481-500. https://doi.org/10.1080/13576500902958871

Koo, T. K., \& Li, M. Y. (2016). A Guideline of Selecting and Reporting Intraclass Correlation Coefficients for Reliability Research. Journal of Chiropractic Medicine, 15(2), 155-63. https://doi.org/10.1016/j.jcm.2016.02.012 
Króliczak, G., Gonzalez, C. L., \& Carey, D. P. (2019). Editorial: Manual Skills, Handedness, and the Organization of Language in the Brain. Frontiers in Psychology. 10:930. https://doi.org/10.3389/fpsyg.2019.00930

Leppanen, M. L., Lyle, K. B., Edlin, F. M., \& Schäfke, V. D. (2018). Is self-report a valid measure of unimanual object-based task performance? Laterality: Asymmetries of Body, Brain and Cognition, 23: 1-21. https://doi.org/10.1080/1357650X.2018.1550493

Mazoyer, B, Zago, L, Jobard, G, Crivello, F, Joliot, et al. (2014). Gaussian Mixture Modeling of Hemispheric Lateralization for Language in a Large Sample of Healthy Individuals Balanced for Handedness. PLoS ONE, 9(6), e101165. https://doi.org/10.1371/journal.pone.0101165

McManus, I. C. (1983). The Interpretation of Laterality. Cortex, 19(2), 187-214. https://doi.org/10.1016/S0010-9452(83)80014-8

McManus, I. C. (1984). The Power of a Procedure for Detecting Mixture Distributions in Laterality Data. Cortex, 20(3), 421-426. https://doi.org/10.1016/S0010-9452(84)80010-6

McManus, I. C. (1985). Right- and left-hand skill: Failure of the right shift model. British Journal of Psychology, 76(1), 1-16. https://doi.org/10.1111/j.2044-8295.1985.tb01926.x

McManus, I. C., Van Horn, J. D., \& Bryden, P. J. (2016). The Tapley and Bryden test of performance differences between the hands: The original data, newer data, and the relation to pegboard and other tasks. Laterality: Asymmetries of Body, Brain and Cognition, 21(46), 371-396. https://doi.org/10.1080/1357650X.2016.1141916

Mellet, E, Jobard, G, Zago, L, Crivello, F, Petit, L, et al. (2014). Relationships between hand laterality and verbal and spatial skills in 436 healthy adults balanced for handedness. Laterality: Asymmetries of Body, Brain and Cognition, 19(4), 383-404.

Milenkovic, S., \& Dragovic, M. (2013). Modification of the Edinburgh Handedness Inventory: A replication study, 18(3), 340-348. https://doi.org/10.1080/1357650X.2012.683196

Newman, S. D., Malaia, E., \& Seo, R. (2014). Does degree of handedness in a group of righthanded individuals affect language comprehension? Brain and Cognition 86, 98-103.

Oldfield, R. C. (1971). The assessment and analysis of handedness: the Edinburgh inventory. Neuropsychologia, 9(1), 97-113. Retrieved from http://www.ncbi.nlm.nih.gov/pubmed/5146491

Pritchard, E., Propper, R. E., \& Christman, S. D. (2013). Degree of handedness, but not direction, is a systematic predictor of cognitive performance. Frontiers in Psychology 4.

R Core Team (2018). R: A Language and Environment for Statistical Computing. $R$ Foundation for Statistical Computing, Vienna, Austria. https://www.R-project.org/

Reuben, D. B., Magasi, S., McCreath, H. E., Bohannon, R. W., Wang, Y.-C., Bubela, D. J., ... Gershon, R. C. (2013). Motor assessment using the NIH Toolbox. Neurology, 80(11 Suppl 3), S65-75. https://doi.org/10.1212/WNL.0b013e3182872e01

Somers, M., Aukes, M. F., Ophoff, R. A., Boks, M. P., Fleer, W., de Visser, K. (C ). L., ... Sommer, I. E. (2015). On the relationship between degree of hand-preference and degree of language lateralization. Brain and Language. https://doi.org/10.1016/j.band1.2015.03.006

Tapley, S. M., \& Bryden, M. P. (1985). A group test for the assessment of performance between the hands. Neuropsychologia, 23(2), 215-221. https://doi.org/10.1016/00283932(85)90105-8

Veale, J. F. (2014). Edinburgh Handedness Inventory - Short Form: A revised version based on confirmatory factor analysis. Laterality: Asymmetries of Body, Brain and Cognition, 19(2), 164-177. https://doi.org/10.1080/1357650X.2013.783045 
Van Essen, D. C., Smith, S. M., Barch, D. M., Behrens, T. E. J., Yacoub, E., Ugurbil, K., \& WUMinn HCP Consortium (2013). The WU-Minn Human Connectome Project: an overview. NeuroImage, 80, 62-79. https://doi.org/10.1016/j.neuroimage.2013.05.041

Van Horn, J. D. (1992). Brain structural abnormality and laterality in schizophrenia (Unpublished $\mathrm{PhD}$ thesis). University College London.

Vingerhoets, G. (2014). Praxis, language, and handedness: a tricky triad. Cortex 57, 294-296. doi: 10.1016/j.cortex.2014.01.019

Wang, Y. C., Magasi, S. R., Bohannon, R. W., Reuben, D. B., McCreath, H. E., Bubela, D. J., Gershon, R. C., \& Rymer, W. Z. (2011). Assessing dexterity function: A comparison of two alternatives for the NIH toolbox. Journal of Hand Therapy, 24(4), 313-321. https://doi.org/10.1016/j.jht.2011.05.001

Willems, R. M., Der Haegen, L. Van, Fisher, S. E., \& Francks, C. (2014). On the other hand: Including left-handers in cognitive neuroscience and neurogenetics. Nature Reviews Neuroscience. https://doi.org/10.1038/nrn3679

Zago, L, Petit, L, Mellet, E, Jobard, G, Crivello, F, et al. (2016). The association between hemispheric specialization for language production and for spatial attention depends on left-hand preference strength. Neuropsychologia, 93, 394-406. https://doi.org/10.1016/j.neuropsychologia.2015.11.018 